\title{
Isolement et quelques caractéristiques des bactériophages de Streptococcus thermophilus et de Lactobacillus helveticus de ferments d'Emmental
}

\author{
par \\ T. SOZZI et R. MARET \\ Département de développement technologique \\ Société d'Assistance technique pour produits Nestlé S.A. \\ Laboratoire Industriel Orbe - 1350 Orbe (Suisse) \\ avec la collaboration technique de Robert POUSAZ
}

La fabrication du fromage Emmental pendant ces dernières années, comme toutes les technologies des autres fromages, a subi de profondes modifications. La plus importante est l'utilisation de cultures pures des ferments mais l'emploi de celles-ci a aggravé le problème des infections phagiques.

Les ferments que l'on trouve dans le commerce pour la production du fromage Emmental sont composés de souches de Streptococcus thermophilus et de Lactobacillus helveticus. Ces microorganismes thermophiles, utilisés dans les pays européens, soit pour la fabrication de plusieurs fromages typiques, soit pour la préparation des yoghourts, sont mal connus, leurs bactériophages et les problèmes qu'ils causent à l'industrie laitière le sont encore moins.

La seule étude publiée sur les phages des ferments d'Emmental est celle de Kiuru et Tybeck [8], lesquels ont isolé de ferments défectueux des phages de Streptococcus thermophilus, de Lactobacillus lactis et de Lactobacillus helveticus.

Deane et al. [2] ont isolé un phage de Streptococcus thermophilus après avoir étudié plus de 50 échantillons de petit-lait provenant de différentes usines.

Plus nombreux sont les phages de Streptococcus thermophilus isolés de cultures de yoghourt $[1,5,7,10]$.

Nous avons isolé plusieurs phages de cultures de Streptococcus thermophilus utilisées pour la fabrication de fromages à pâte molle (Bel Paese et type Mozzarella) [13] et un des ferments de Gorgonzola. 
Nous avons également trouvé un phage de Lactobacillus lactis de ce ferment [12].

En étudiant les causes de mauvaise acidification de la fabrication d'Emmental dans une usine de production, nous avons isolé des phages spécifiques de Streptococcus thermophilus et de Lactobacillus helveticus dont on donne les caractéristiques dans cette publication.

\section{METHODES}

\section{Souches bactériennes}

Les Streptococcus thermophilus

S265 isolé de la culture du commerce utilisée en usine.

L 12, 153, 24, R2, S110, S 19, YS 1, YS2, YS3, YS4, YS5, YS6, YS7, YS 8, YS9, YS 10, YS11, YS 12 de notre collection.

L76, reçu de la Station fédérale Liebefeld-Berne.

440, 446, 447 reçus de M. J. P. Accolas, I.N.R.A. Jouy-en-Josas.

Les Lactobacillus helveticus

L112 isolé de la culture du commerce utilisé en fabrique.

$\mathrm{H}_{2}$, Ro151 reçus du professeur V. Bottazzi, Université de Piacenza.

L77, L 78 reçus de la Station fédérale Liebefeld-Berne.

15807 A.T.C.C.

\section{Phages}

Lactobacillus helveticus

15807 A.T.C.C. (ex Kiuru et al.).

$\mathrm{FiLH}_{3}$ reçu de M. J.P. Accolas, I.N.R.A., Jouy-en-Josas.

Streptococcus thermophilus

FiLST 5

FiLST 8$\}$ reçus de M. J. P. Accolas, I.N.R.A., Jouy-en-Josas.

19987 A.T.C.C. (ex. Kiuru et Tybeck).

$24, \mathrm{~s} 19, \mathrm{r}, 112, \mathrm{~s} 133, \mathrm{~s} 110$ de notre collection.

\section{Milieux}

- Lait écrémé reconstitué à 10 p. $100+0,1$ p. 1000 d'extrait de levure, stérilisé à $115^{\circ} \mathrm{C}$ pendant $30 \mathrm{mn}$.

- MRS [3] pour les lactobacilles et leurs phages.

- Hogg et Jago [6] pour les souches hôtes et le phage de streptocoque. 


\section{Dénombrement des phages}

Deux méthodes sont employées :

1) Plages de lyse selon Reinbold [11] avec les milieux cités précédemment.

2) Dénombrement selon Valles [16] avec contrôle de la présence de phages dans les trois premières dilutions coagulées.

\section{Morphologie}

Les observations et la photo sont faites au microscope électronique Philips EM 300 selon Dentan et al. [4].

\section{Résistance à la chaleur}

On a utilisé la méthode de Kiuru [8] mais à ces températures :

$55^{\circ} \mathrm{C}$ température de chauffage du fromage d'Emmental.

$65^{\circ} \mathrm{C}$ température de pasteurisation utilisée dans les fromageries artisanales.

$72^{\circ} \mathrm{C}$ température de pasteurisation employée dans les productions industrialisées.

$90^{\circ} \mathrm{C}$ température de pasteurisation du lait utilisé pour la préparation des ferments.

Les échantillons sont prélevés après: $20 \mathrm{~s} / 5 \mathrm{mn} / 10 \mathrm{mn} / 15 \mathrm{mn} /$ $30 \mathrm{mn}$ et $1 \mathrm{~h}$.

\section{Détermination de la spécificité}

On a utilisé les cultures sur lait selon Sozzi [13].

\section{Température de développement}

Un appareil, comme décrit par Oppenheimer [9] a servi pour les essais.

Deux séries de 25 tubes contenant $10 \mathrm{ml}$ de milieu sont équilibrées pendant $12 \mathrm{~h}$ à une température de $20^{\circ} \mathrm{C}$ à $55^{\circ} \mathrm{C}$ et inoculées avec $0,1 \mathrm{ml}$ de culture sur bouillon à $\sim 10^{7}$ c.f.u. $/ \mathrm{ml}$ (colony forming units) et $0,1 \mathrm{ml}$ de suspension de phages à $10^{\circ}$ p.f.u. $/ \mathrm{ml}$ (plaque forming units).

Après $6 \mathrm{~h}$ et $9 \mathrm{~h}$ pour le phage du lactobacille et après $3 \mathrm{~h}$ et $6 \mathrm{~h}$ pour le phage du streptocoque, on a évalué la D.O. au spectrophotomètre et déterminé le développement des phages par la lyse de la culture. 


\section{Résistance au pH}

Le contenu de biberons de $100 \mathrm{ml}$ de milieu est réglé exactement au $\mathrm{pH}$ suivant, avec $\mathrm{NaOH} / \mathrm{N}$ ou $\mathrm{HCl} / \mathrm{N}$ : $\mathrm{pH} 3$ - 4 - 4,5 - 5 - 5,5 - 6 $6,5-7-8-9-10-11$.

A chaque biberon on ajoute $1 \mathrm{ml}$ de suspension de phages $\left(\sim 10^{9}\right.$ p.f.u. $\left./ \mathrm{ml}\right)$ et après $20 \mathrm{~s}, 5 \mathrm{mn}, 30 \mathrm{mn}, 1 \mathrm{~h}, 6 \mathrm{~h}, 24 \mathrm{~h}$ et $7 \mathrm{j}$ on prélève $1 \mathrm{ml}$ que l'on met dans $10 \mathrm{ml}$ de lait avant de faire les dilutions en solution de Ringer pour déterminer les phages restants.

\section{pH de développement}

Deux séries de tubes avec $10 \mathrm{ml}$ de bouillon, $\mathrm{pH} 3-4-4,5$ - 5 5,5 - 6 - 6,5 sont inoculées avec $0,1 \mathrm{ml}$ de culture de la souche hôte et, à une série, on ajoute $0,1 \mathrm{ml}$ d'une suspension de phages $\left(10^{\circ}\right.$ p.f.u./ml).

Après $6 \mathrm{~h}$ on lit le développement bactérien au spectrophotomètre, la multiplication des phages est observée par la lyse des cellules bactériennes dans les tubes infectés.

\section{Action de I'hypochlorite}

Des tubes contenant $10 \mathrm{ml}$ de solution standard d'hypochlorite de $\mathrm{Na}$ sont additionnés de $1 \mathrm{ml}$ de suspension de phages $\left(10^{\circ}\right.$ p.f.u./ml); après $20 \mathrm{~s}, 1 \mathrm{mn}, 5 \mathrm{mn}, 30 \mathrm{mn}$ on prélève $1 \mathrm{ml}$ que l'on met dans $10 \mathrm{ml}$ de lait stérile et on titre le nombre de phages survivants.

\section{Exigence en calcium}

On a utilisé la méthode de Sozzi [15].

\section{RESULTATS}

L'isolement du phage de Streptococcus thermophilus a été facile, on a pu constater sa présence directement dans les cultures d'Emmental, après adjonction de filtrat d'une culture mal acidifiée ou de filtrats de trois échantillons de sérum différents, par la disparition de tous les coques.

La purification du phage est faite sur la souche S265 de Streptococcus thermophilus et on l'a, pour cela, appelé s 265.

Le phage de Lactobacillus helveticus appelé 1112 a été trouvé seulement dans les échantillons de sérum après enrichissement sur la souche L112 de Lactobacillus helveticus. 
Le test de la spécificité fait sur 23 souches de Streptococcus thermophilus de différentes provenances a démontré qu'une seule souche, YS4, est sensible à ce phage en plus de son hôte S265.

De la souche YS4, isolée d'une culture de yoghourt, on ne possède pas de phage.

La souche S265 n'est lysée par aucun phage de Streptococcus thermophilus de notre collection.

La spécificité du phage 1112 est nette, celui-ci ne lyse aucune des souches testées de Lactobacillus helveticus de notre collection.

La souche L112, en outre, n'est pas lysée par les phages spécifiques de Lactobacillus helveticus de notre collection.

\section{Résistance à la chaleur}

Les courbes de destruction du phage s265 par la chaleur en fonction du temps sont représentées dans la figure 1. Le phage résiste à $55^{\circ} \mathrm{C}$ pendant toute la durée de l'essai, à $65^{\circ} \mathrm{C}$ on voit une diminution progressive en fonction du temps et on arrive à la complète destruction après $30 \mathrm{mn}$.

Si le phage est maintenu à $72^{\circ} \mathrm{C}$ la diminution des particules phagiques est plus rapide et après $10 \mathrm{mn}$ toutes sont inactives.

La température de $90^{\circ} \mathrm{C}$ est fatale pour ce phage, après $20 \mathrm{~s}$ on ne retrouve aucun survivant.

La figure 2 montre les courbes de survie, aux différentes températures étudiées, du phage 1112 . Ce phage est parfaitement stable à $55^{\circ} \mathrm{C}$ pendant les premières $30 \mathrm{mn}$ mais dans les $30 \mathrm{mn}$ suivantes on remarque une légère baisse de $10^{\circ}$ à $10^{7}$.

La température de $65^{\circ} \mathrm{C}$ provoque une forte mortalité des phages, après $20 \mathrm{~s}$ on a déjà une réduction de $10^{\circ}$ à $10^{5}$, la survie à $5 \mathrm{mn}$ est de $10^{3}$ et reste stable pendant les $25 \mathrm{mn}$ suivantes pour arriver, après $60 \mathrm{mn}$, à $10^{2}$.

La réduction des virus est plus forte à $72^{\circ} \mathrm{C}$. On commence avec $10^{9}$ pour arriver en $20 \mathrm{~s}$ à $10^{4}, 10^{3}$ en $5 \mathrm{mn}$ et 10 après $10 \mathrm{mn}$ et $30 \mathrm{mn}$. On ne retrouve plus de survivants après $60 \mathrm{mn}$.

La température de $90^{\circ} \mathrm{C}$ est mortelle déjà après $20 \mathrm{~s}$.

\section{Température de développement}

Les résultats de ces essais sont reportés dans les figures 3 et 4 .

Le résultat obtenu avec le streptocoque S 265 et son phage est présenté dans la figure 3 qui montre une étroite relation entre le développement de la bactérie et celui du phage. Le streptocoque se développe progressivement d'une façon régulière à partir de $25^{\circ} \mathrm{C}$ et le maximum est atteint à $38^{\circ} \mathrm{C}$, suivi d'une baisse rapide jusqu'à $45^{\circ} \mathrm{C}$; au-delà de cette température le développement est bloqué. 


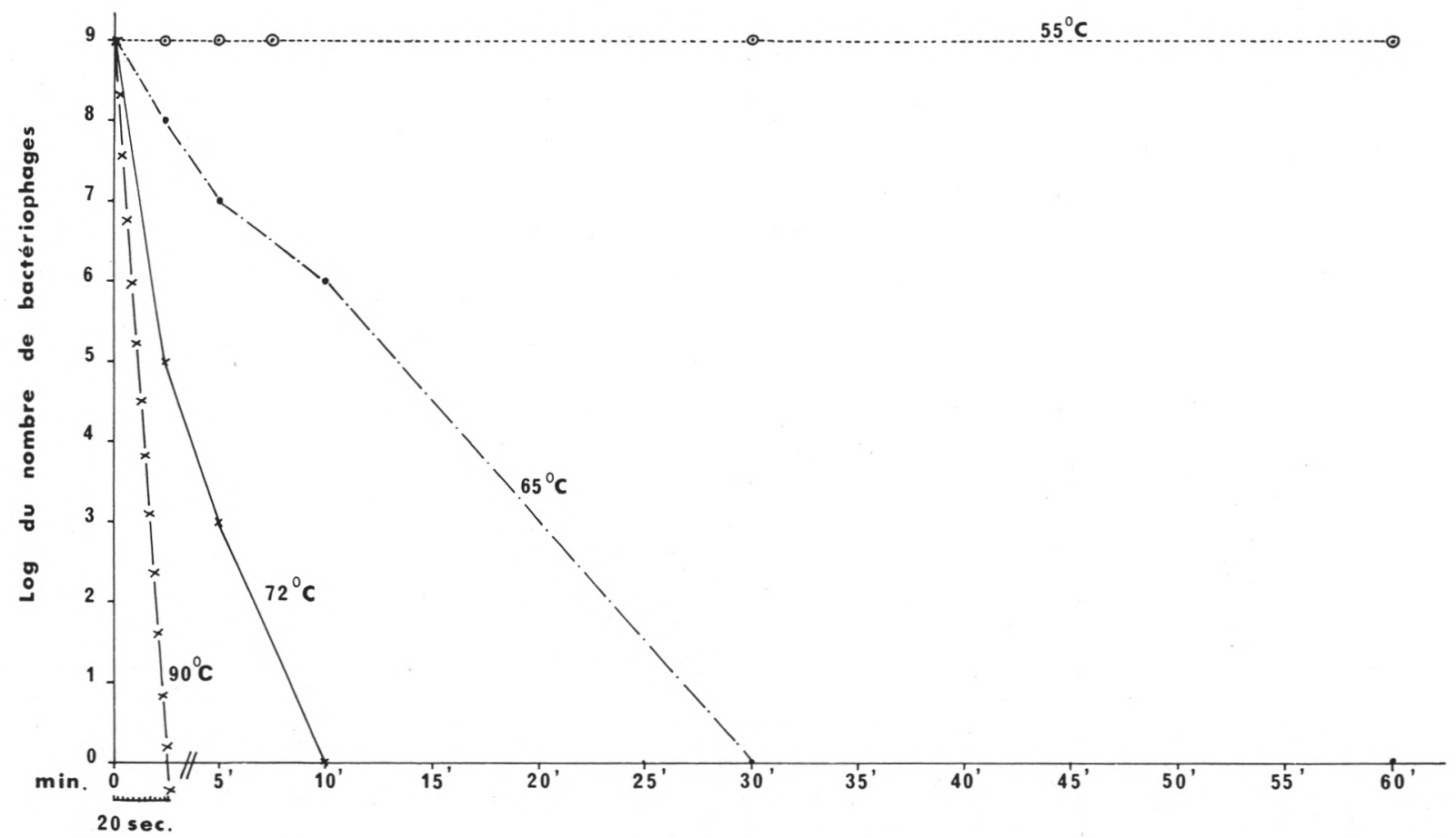

fig. 1

Destruction du phage s 265 par la chaleur à différentes températures en fonction du temps 


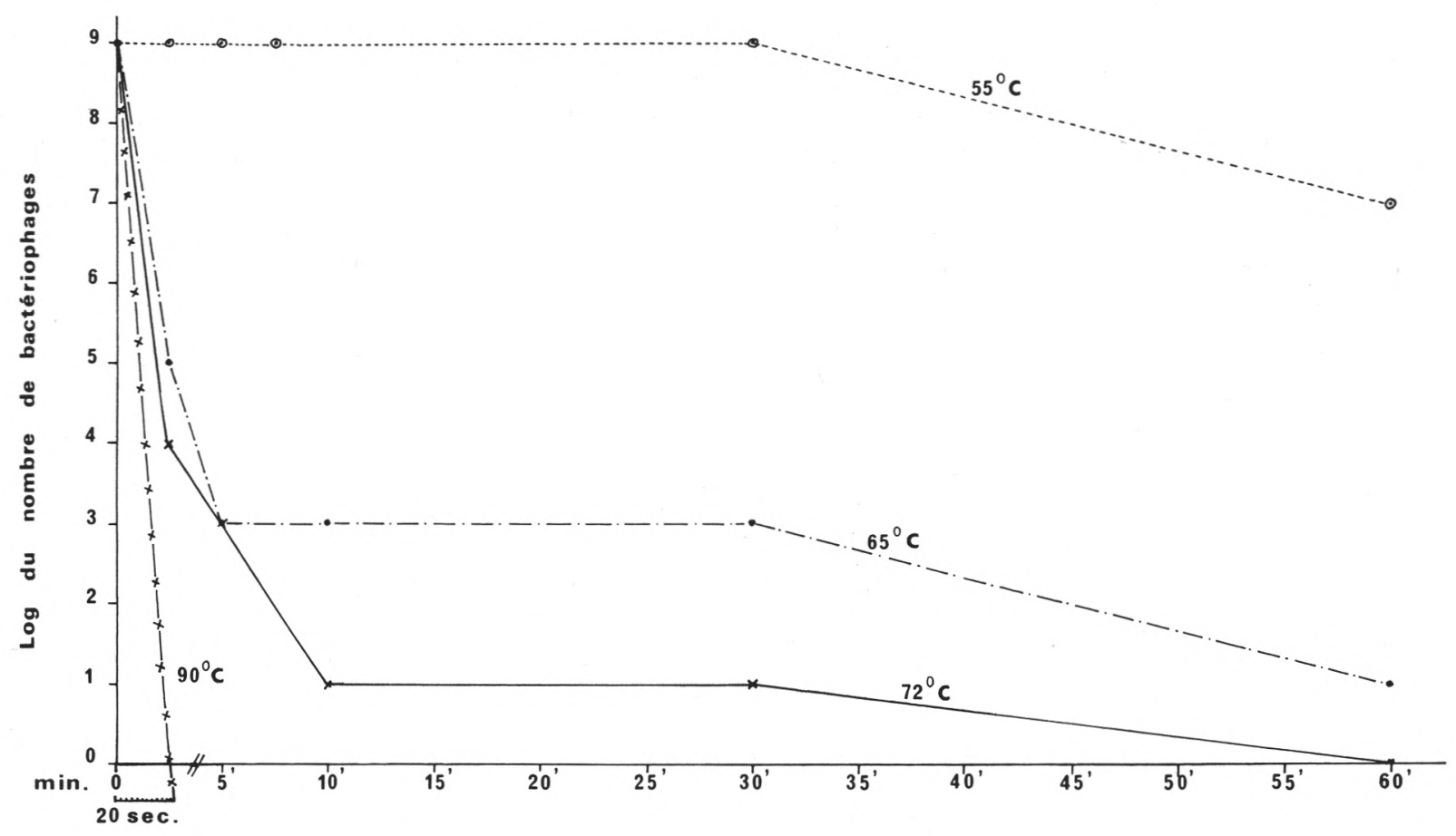

fig. 2

Destruction du phage 1112 par la chaleur à différentes températures en fonction du temps 


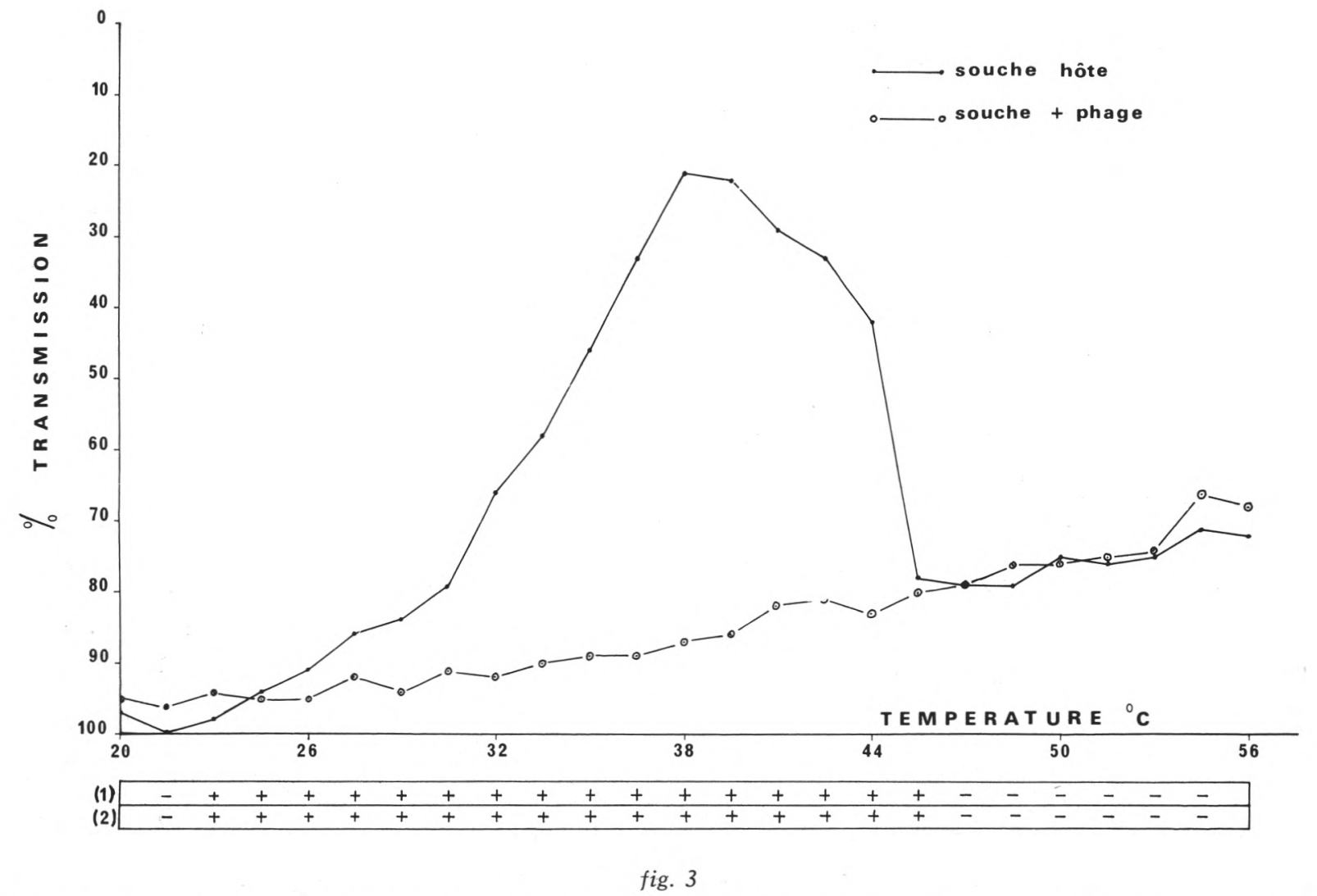

Multiplication du Str. thermophilus S 265 et de son phage s 265 en fonction des températures d'incubation pendant $6 \mathrm{~h}$

(1) Observation visuelle de la culture $\quad\{+=$ développement

(2) Observation visuelle de la culture + phage $\{-=$ pas de développement 
La lyse phagique se manifeste à toutes les températures auxquelles les cultures se développent.

L'augmentation de la température entraîne un certain brunissement du milieu et, par conséquent, une diminution de la transmission de la lumière.

Dans la figure 4 sont rapportées les courbes de développement du Lactobacillus helveticus L112 et de son phage 1112, en fonction de la température. On voit que les bactéries ne se développent pas à $20^{\circ} \mathrm{C}$ mais, par contre, sont à leur maximum entre $35^{\circ} \mathrm{C}$ et $45^{\circ} \mathrm{C}$. Elles se multiplient moins bien jusqu'à $50^{\circ} \mathrm{C}$. Le phage 1112 ne lyse la culture qu'entre $30^{\circ} \mathrm{C}$ et $47^{\circ} \mathrm{C}$.

L'adjonction de la préparation de phages en dehors des températures de son développement, a une action stimulante sur la multiplication bactérienne.

\section{Influence du pH}

\section{Destruction des phages}

Les résultats du tableau 1 montrent que le phage s 265 est détruit s'il est conservé à $\mathrm{pH} 3 \geqslant 10 \mathrm{mn}$ et à $\mathrm{pH} 11 \geqslant 60 \mathrm{mn}$; sa stabilité est intacte à toutes les autres valeurs de $\mathrm{pH}$, même après $7 \mathrm{j}$.

Comme on l'observe dans le tableau 2, le phage 1112 survit très bien à tous les $\mathrm{pH}$ essayés entre 3 et 11 pendant les premières $24 \mathrm{~h}$ et c'est seulement après 7 j qu'il est détruit à $\mathrm{pH} 3$ et $\mathrm{pH} 11$.

\section{Développement des phages}

Comme démontré dans la figure 5 le phage s 265 ne peut se multiplier à $\mathrm{pH}$ inférieur à 5 , la culture commence à se développer à $\mathrm{pH} \mathrm{4,5}$.

La figure 6 donne les résultats de la multiplication du phage 1112 , qui commence à $\mathrm{pH} \geqslant 4,5$. Le développement de la culture se manifeste faiblement à pH 3 avec une certaine augmentation jusqu'à 4,5.

\section{Exigence en calcium}

La présence du calcium s'est révélée indispensable pour le phage s265; au contraire, le phage de Lactobacillus 1112 se multiplie parfaitement dans le milieu MRS sans adjonction de chlorure de calcium après plusieurs repiquages.

\section{Influence du chlore}

Les phages s 265 et 1112 survivent au moins $30 \mathrm{mn}$ dans une solution contenant 0,01 p. 100 de chlore actif (voir tab. 3 et 4). Avec une solution à 0,05 p. 100 de chlore actif ils sont fortement réduits entre $20 \mathrm{~s}$ et $1 \mathrm{mn}$ de contact et complètement détruits après $5 \mathrm{mn}$. 
TABLEAU 1

Inactivation du phage s 265 à différentes valeurs de $\mathrm{pH}$ en fonction du temps

\begin{tabular}{|c|c|c|c|c|c|c|c|c|c|c|c|c|}
\hline Temps & pH 3 & pH 4 & $\mathrm{pH} 4,5$ & pH 5 & pH 5,5 & pH 6 & $\mathrm{pH} 6,5$ & $\mathrm{pH} 7$ & $\mathrm{pH} 8$ & $\mathrm{pH} 9$ & $\mathrm{pH} 10$ & $\mathrm{pH} 11$ \\
\hline $20 \mathrm{~s}$ & $8(1)$ & 8 & 7 & 8 & 8 & 7 & 7 & 6 & 9 & 10 & 8 & 7 \\
\hline $10 \mathrm{mn}$ & 0 & 8 & 8 & 8 & 8 & 8 & 8 & 7 & 8 & 7 & 8 & 4 \\
\hline $30 \mathrm{mn}$ & 0 & 8 & 7 & 7 & 7 & 8 & 8 & 7 & 8 & 8 & 8 & 4 \\
\hline $60 \mathrm{mn}$ & 0 & 8 & 8 & 7 & 7 & 8 & 8 & 7 & 8 & 8 & 8 & 0 \\
\hline $6 \mathrm{~h}$ & 0 & 8 & 8 & 7 & 7 & 8 & 8 & 7 & 8 & 8 & 8 & 0 \\
\hline $24 \mathrm{~h}$ & 0 & 7 & 8 & 8 & 7 & 8 & 7 & 8 & 9 & 7 & 7 & 0 \\
\hline $7 \mathrm{j}$ & 0 & 7 & 7 & 8 & 8 & 8 & 8 & 7 & 7 & 6 & 6 & 0 \\
\hline
\end{tabular}

(1) log. du nombre de phages. 
TABLEAU 2

Inactivation du phage 1112 à différentes valeurs de $\mathrm{pH}$ en fonction du temps

\begin{tabular}{|c|c|c|c|c|c|c|c|c|c|c|c|c|}
\hline Temps & pH 3 & $\mathrm{pH} 4$ & $\mathrm{pH} 4,5$ & pH 5 & $\mathrm{pH} \mathrm{5,5}$ & pH 6 & $\mathrm{pH} 6,5$ & pH 7 & $\mathrm{pH} 8$ & pH 9 & $\mathrm{pH} 10$ & pH 11 \\
\hline $20 \mathrm{~s}$ & $6(1)$ & 6 & 7 & 7 & 7 & 7 & 7 & 6 & 6 & 5 & 6 & 5 \\
\hline $10 \mathrm{mn}$ & 6 & 6 & 7 & 7 & 7 & 7 & 6 & 6 & 6 & 5 & 6 & 5 \\
\hline $30 \mathrm{mn}$ & 5 & 7 & 6 & 7 & 6 & 6 & 6 & 6 & 6 & 6 & 7 & 5 \\
\hline $60 \mathrm{mn}$ & 5 & 6 & 7 & 7 & 7 & 8 & 5 & 6 & 6 & 6 & 6 & 4 \\
\hline $6 \mathrm{~h}$ & 4 & 5 & 6 & 5 & 7 & 7 & 5 & 6 & 5 & 6 & 4 & 4 \\
\hline $24 \mathrm{~h}$ & 5 & 5 & 6 & 5 & 7 & 5 & 5 & 6 & 5 & 6 & 5 & 3 \\
\hline $7 \mathrm{j}$ & 0 & 6 & 5 & 5 & 5 & 6 & 5 & 5 & 6 & 5 & 3 & 0 \\
\hline
\end{tabular}

(1) log. du nombre de phages. 


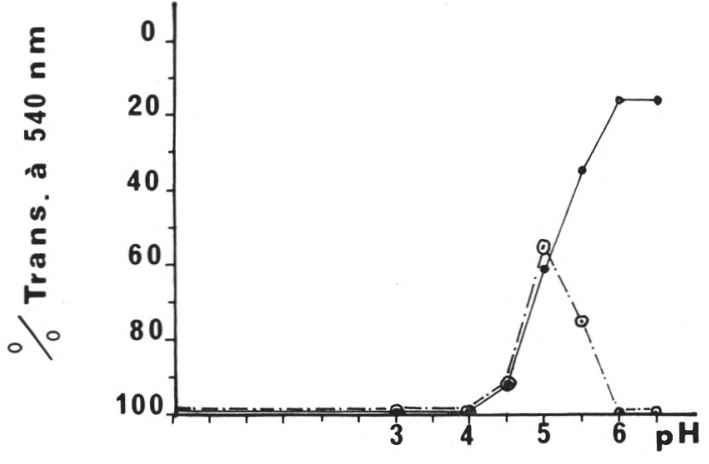

fig. 5

Développement du Str. thermophilus S265 et de son phage s265 en fonction du pH après $5 \mathrm{~h} 30 \mathrm{mn}$ d'incubation.

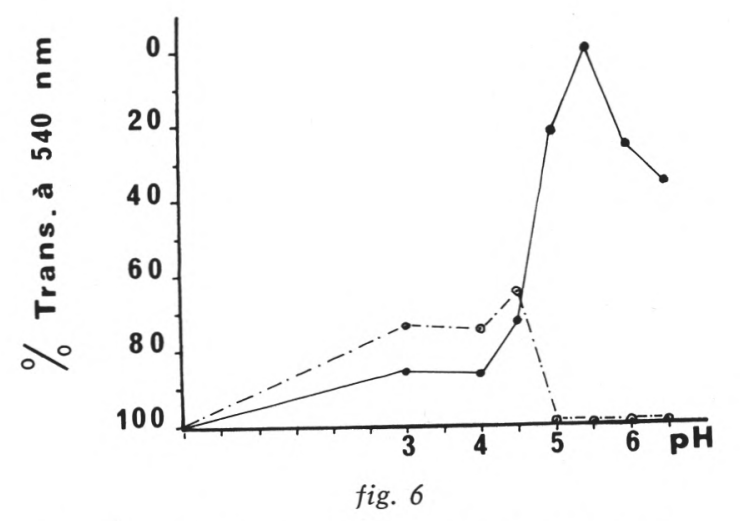

Développement du L. helveticus $\mathrm{L} 112$ et de son phage 1112 en fonction du pH après $5 \mathrm{~h} 30 \mathrm{mn}$ d'incubation. 
TABLEAU 3

Destruction du phage s 265 par différentes concentrations de chlore en fonction du temps

\begin{tabular}{c|c|c|c}
\hline Conc. p. 100 & 0,05 & 0,01 & 0,005 \\
\hline & & & \\
$20 \mathrm{~s}$ & $3(1)$ & 7 & 9 \\
$1 \mathrm{mn}$ & 2 & 7 & 9 \\
$5 \mathrm{mn}$ & 0 & 7 & 9 \\
$30 \mathrm{mn}$ & 0 & 7 & 9 \\
\hline
\end{tabular}

(1) log. du nombre de phages.
TABLEAU 4

Destruction du phage 1112 par différentes concentrations de chlore en fonction du temps

\begin{tabular}{c|c|c|c}
\hline Conc. p. 100 & 0,05 & 0,01 & 0,005 \\
\hline & & & \\
$20 \mathrm{~s}$ & $3(1)$ & 6 & 7 \\
$1 \mathrm{mn}$ & 3 & 6 & 7 \\
$5 \mathrm{mn}$ & 0 & 6 & 7 \\
$30 \mathrm{mn}$ & 0 & 5 & 6
\end{tabular}

(1) log. du nombre de phages. 


\section{Morphologie}

La morphologie du phage s265 (photo 1) est semblable à celle des phages de Streptococcus thermophilus que nous avons étudiés et à celle du phage 19987 de A.T.C.C.

On peut le classer dans le groupe B de Bradley.

Le phage 1112 (photo 2) montre une morphologie non typique des phages des ferments lactiques car elle présente un manchon le long de la queue qui semble contractile. Sa morphologie est similaire à celle des phages de la souche de Lactobacillus helveticus 15807 A.T.C.C. (note en cours de préparation).

\section{DISCUSSION}

L'isolement de ces deux phages a de nouveau démontré la validité de notre théorie ; la multiplication en très grande quantité d'un microorganisme produit inévitablement l'apparition des phages spécifiques.

On ne peut pas utiliser longtemps les mêmes souches dans une fromagerie sans problèmes de phages.

Le phage de Streptococcus thermophilus a été isolé dans un ferment peu acidifié de même que dans trois échantillons de petit-lait. Celui de Lactobacillus helveticus a pu être isolé seulement dans des échantillons de petit-lait. Ceci est dû aux différentes vitesses de croissance des deux micro-organismes. Dans les 2 premières heures, seuls les streptocoques se multiplient, et c'est après seulement que les lactobacilles croissent ; par conséquent les phages des lactobacilles apparaissent beaucoup plus tard que ceux des streptocoques.

La spécificité des deux phages est très étroite. Des trente souches testées une seule est sensible en plus des deux souches hôtes. Une rotation des souches microbiennes pourrait éliminer les défauts causés par les phages.

La destruction de ces deux phages par la chaleur est possible à une température supérieure à celle utilisée pour la pasteurisation du lait dans les industries laitières.

Le chauffage à $90^{\circ} \mathrm{C}$ élimine tous les phages. Par conséquent, si on veut des ferments exempts de phages il faut, après chauffage du lait à $90^{\circ} \mathrm{C}$, empêcher toute infection phagique ultérieure. Il faut que l'air soit pauvre en phages, condition qui s'applique également aux appareils et outils et que l'on obtient par nettoyage et désinfection. Les valeurs de destruction des phages par la chaleur, trouvées par nous, sont assez proches de celles publiées par les autres chercheurs. 


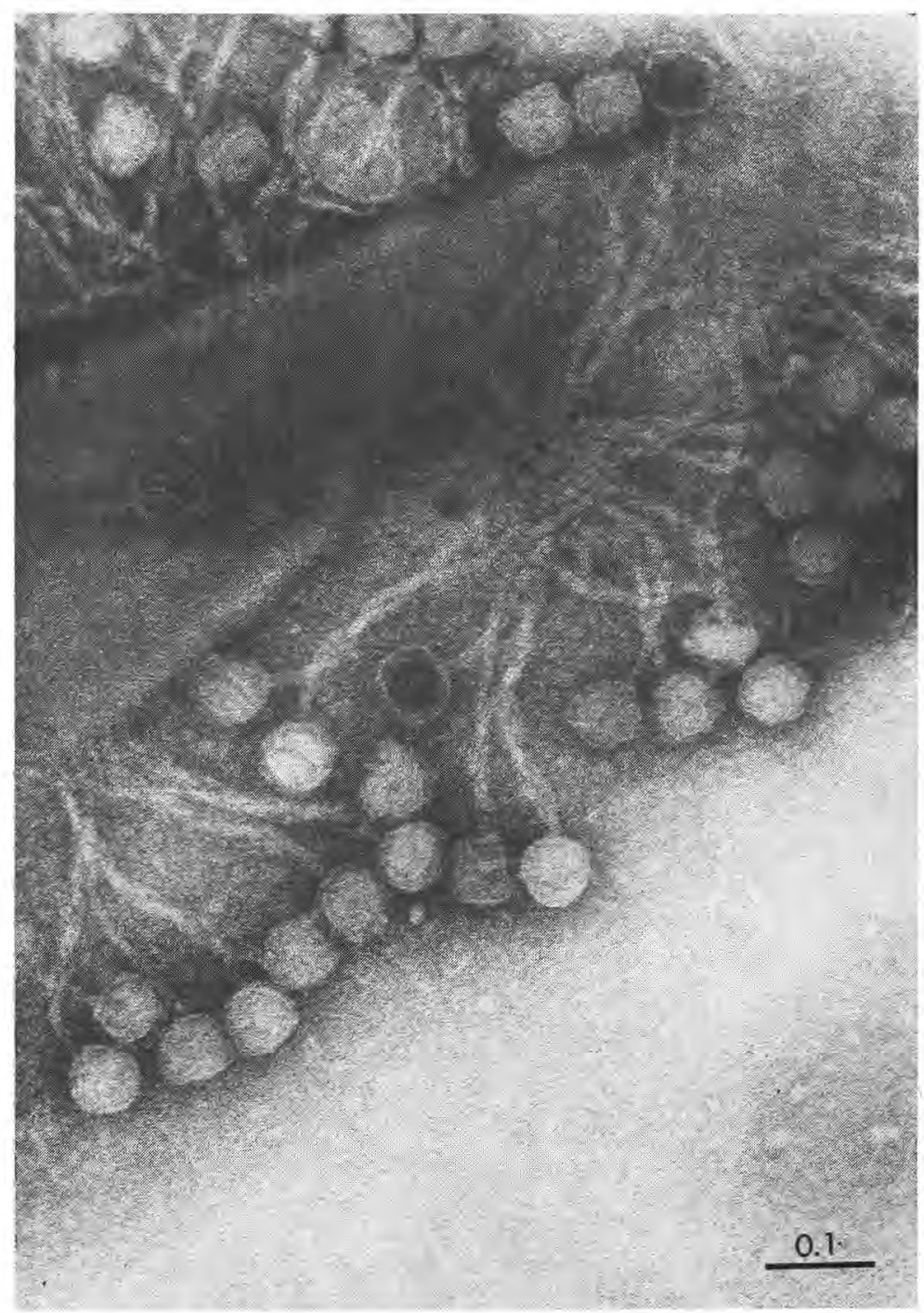

photo 1

Morphologie du phage de Streptococcus thermophilus s 265. Echelle en microns. 


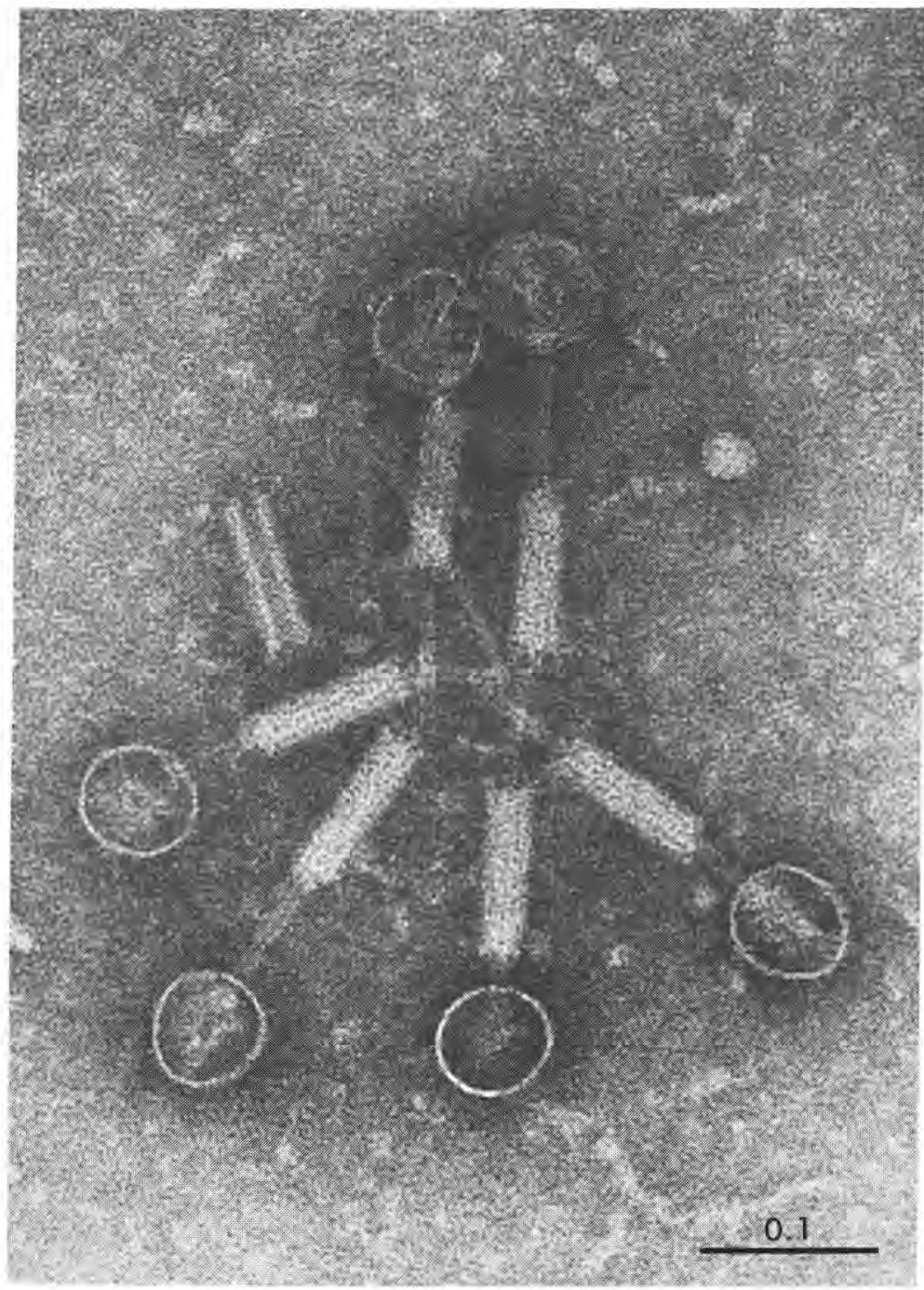

\section{photo 2}

Morphologie du phage de Lactobacillus helveticus L112.

Echelle en microns. 
La courbe de développement en fonction de la température montre qu'il est impossible de limiter le développement du phage s 265 sans limiter celui des hôtes.

Pour le phage 1112 on peut limiter sa multiplication à $>30^{\circ} \mathrm{C}$ mais le lactobacille croît trop lentement à cette température.

L'utilisation des solutions à $\mathrm{pH}$ extrêmes pour détruire les phages en question est à écarter. Ils résistent davantage à des valeurs de pH extrêmes que les phages isolés par Kiuru et Tybeck [8].

L'action du chlore est nette et il suffit d'une concentration de 0,05 p. 100 pour détruire tous les phages, ceci est très important pour désinfecter l'air et les équipements dans les usines. Les valeurs trouvées confirment celles données par d'autres chercheurs.

L'écart de 0,5 pH entre le développement des phages et des souches hôtes ne peut pas être utilisé pour limiter la multiplication parce que les bactéries poussent trop lentement à $\mathrm{pH}$ bas. En $4 \mathrm{~h}$ à $\mathrm{pH} 6$ elles atteignent le même nombre de cellules qu'en $24 \mathrm{~h}$ à $\mathrm{pH} 4,5$ ou $\mathrm{pH}$ 5. Ceci explique pourquoi les phages de lactobacilles sont peu nombreux dans les cultures mixtes de yoghourt ou d'Emmental où les streptocoques poussent plus vite et abaissent le $\mathrm{pH}$ à 4,5 avant que les lactobacilles ne se soient multipliés et, par conséquent, leurs phages sont inhibés.

La différence d'exigence en calcium des deux phages rend inutile l'utilisation des milieux spéciaux pauvres en $\mathrm{Ca}$ pour limiter leur développement dans les fromageries.

La morphologie différente des deux phages facilite la compréhension de la spécificité des phages de Streptococcus thermophilus et Lactobacillus helveticus ; chaque souche a donc son phage propre.

Il faut souligner que la morphologie des phages de Streptococcus thermophilus étudiée par nous a toujours été pareille indépendamment des lieux et des périodes de leur isolement.

La structure du phage de Lactobacillus helveticus 1112, même avec des dimensions différentes, est similaire à celle du phage de Lactobacillus helveticus 15807 A.T.C.C. On serait tenté de dire que cette morphologie est typique des phages de Lactobacillus helveticus mais notre étude sur les morphologies des autres phages de ferments lactiques et les observations d'autres chercheurs portent à la conclusion qu'il peut exister d'autres phages de ces bactéries avec différentes morphologies.

\section{Remerciements}

Nous remercions $M . H$. Bauer et Mlle E. Dentan pour les photos au microscope électronique, $M$. H. Bohren pour son aide dans la préparation de cette publication et $M$. O. Depierraz pour son assistance technique.

TS/RM/mk/11-11-1974. 


\section{Ré s u m é}

Du petit-lait d'une fabrique d'Emmental deux bactériophages ont été isolés, spécifiques respectivement du Str. thermophilus S265 et du L. helveticus L112. Sur vingt-trois autres souches de Str. thermophilus testées vis-à-vis du phage s 265 , une seule a été trouvée sensible. Sur cinq souches de $L$. helveticus examinées, aucune n'a été lysée par le phage 1112 .

Le phage s 265 est détruit par la chaleur en $30 \mathrm{mn}$ à $65^{\circ} \mathrm{C}$, en $10 \mathrm{mn}$ à $72^{\circ} \mathrm{C}$ et en $20 \mathrm{~s}$ à $90^{\circ} \mathrm{C}$. Le phage 1112 a survécu à $1 \mathrm{~h}$ d'exposition à $65^{\circ} \mathrm{C}$, bien qu'avec une forte diminution en nombre ; il est détruit après $1 \mathrm{~h}$ à $72^{\circ} \mathrm{C}$ et après $20 \mathrm{~s}$ à $90^{\circ} \mathrm{C}$.

Le phage s265 s'est multiplié à toutes les températures auxquelles la souche hôte se développait. Le phage 1112 ne s'est multiplié qu'entre $28^{\circ} \mathrm{C}$ et $45^{\circ} \mathrm{C}$, alors que la zone de croissance de la souche hôte était comprise entre $24^{\circ} \mathrm{C}$ et $50^{\circ} \mathrm{C}$.

A un $\mathrm{pH}<3$ et $>11$ le phage $\mathrm{s} 265$ était détruit en $60 \mathrm{mn}$, tandis qu'aux mêmes valeurs de $\mathrm{pH}$ le phage 1112 ne l'était qu'après $7 \mathrm{j}$. La limite inférieure des valeurs de $\mathrm{pH}$ auxquelles le développement du phage $\mathrm{s} 265$ était possible se situait à $\mathrm{pH} 5$; elle l'était à $\mathrm{pH} 4,5$ pour le phage 1112 .

Le chlore actif à une concentration de 500 ppm détruit les deux phages en $5 \mathrm{mn}$.

Le phage s265 avait besoin de $\mathrm{Ca}^{++}$pour se multiplier. Le phage 1112, au contraire, était tout à fait capable de se propager en l'absence de $\mathrm{Ca}^{++}$.

Le phage s265 est morphologiquement du type B de Bradley; le phage 1112 est du type A.

\section{S u $\mathrm{m} \mathbf{m}$ a r y}

Two bacteriophages specific for Str. thermophilus S265 and $L$. helveticus respectively, were isolated from whey from an Emmental factory. Of 23 other strains of Str. thermophilus tested, only one proved susceptible to phage s265. Of 5 other strains of $L$. helveticus tested, none was lysed by phage 1112 .

Phage s 265 is destroyed by heating at $65^{\circ} \mathrm{C}$ for $30 \mathrm{mn}, 72^{\circ} \mathrm{C}$ for $10 \mathrm{mn}$ and $90^{\circ} \mathrm{C}$ for $10 \mathrm{~s}$. Phage 1112 survived exposure to $65^{\circ} \mathrm{C}$ for 1 hour, though with a large decrease in number ; it is destroyed after 1 hour at $72^{\circ} \mathrm{C}$ and $20 \mathrm{~s}$ at $90^{\circ} \mathrm{C}$.

Phage s265 multiplied at all the growth temperatures of the host strain. Phage 1112 only multiplied between $28^{\circ} \mathrm{C}$ and $45^{\circ} \mathrm{C}$ whereas the host strain grew from $24^{\circ} \mathrm{C}$ to $50^{\circ} \mathrm{C}$. 
Below $\mathrm{pH} 3$ and above $\mathrm{pH} 11$, phage s265 was destroyed in $60 \mathrm{mn}$ while phage 1112 was only destroyed after 7 days. The minimum $\mathrm{pH}$ for growth was 5 for phage s 265 and 4,5 for phage 1112 .

Chlorine at a concentration of 500 ppm kills both phages in $5 \mathrm{mn}$.

Calcium was necessary for the growth of phage s 265 but not for phage 1112.

Phage s265 is morphologically type B of Bradley, phage 1112 is type A.

Reçu pour publication en décembre 1974.

\section{Bibliographie}

[1] CiBLIS (E.) (1966). - Bekämpfung eines Bakteriophagen von Streptococcus thermophilus. XVIII int. Dairy Cong., vol. D, 395-400.

[2] Deane (D. A.), Nelson (F. E.), Ryser (F. C.) and Carr (P. H.) (1953). Streptococcus thermophilus bacteriophage from series cheese whey. J. Dairy Sci., 36, 185-191.

[3] De Man (J. C.), Rogosa (M.) and Sharpe (E.) (1960). - Medium for the cultivation of Lactobacilli. J. Appl. Bact., 23, 130-135.

[4] Dentan (E.), Sozzi (T.) and Bauer (H.) (1970). - The Morphology of two Lactobacillus bacteriophages. J. de Microscopie, 9, 567-570.

[5] Gelin (M.), Wurch (T.) et Linder (R.) (1970). - Mise en évidence d'un bactériophage du Streptococcus thermophilus cause d'un accident de fermentation du yoghourt. C.R. Acad. Sc. Paris, t. 270, sér. D., 425-427.

[6] Hogg (Mc C. D.) and JAGo (G. R.) (1970). - Extraction of the $260 \mathrm{~nm}$ absorbing material from Group N. Streptococci as a method for estimating cell growth. J. Dairy Res., 37, 199-202.

[7] Katrandzhiev (K.) and Stoyanov (S.) (1961). - Bacteriophage in starter for cultured milk and measure to control it. Dairy Sci. Abst., 23, 2641.

[8] KiuRU (V.J.) and TYвесK (E.) (1955). - Characteristics of bacteriophages active against lactic acid bacteria in swiss cheese. Suomen. Kem., 288, $56-62$.

[9] Oppenheimer (C. H.) and Drost-Hansen (W. A.) (1960). - A relationship between multiple temperature optima for biological systems and the properties of water. J. Bact., 80, 21-24.

[10] Pette (J. W.) and Kooy (J.S.) (1952). - Bacteriophage in yogurt. Neth. Milk and Dairy J., 6, 233-241.

[11] Reinbold (G. W.) and REDDy (M. S.) (1973). - Bacteriophage for Streptococcus thermophilus. Dairy Industries, 38, 412-416.

[12] Sozzi (T.) (1966). - Batteriofago di Lactobacillus sp. nell' industria casearia. Ann. Microb., 16, 37-38.

[13] Sozzi (T.) et Prella (G.) (1968). - Specificita batteriofagica in Streptococcus thermophilus. Ann. Micr., 18, 239-242.

[14] Sozzi (T.) et Sella (F.) (1970). - Sulla comparsa di batteriofago attivo verso Streptococcus thermophilus durante la preparazione delle colture per Gorgonzola. Ann. Micr., 20, 73-79.

[15] Sozzi (T.) (1972). - Etude sur l'exigence en calcium des phages des ferments lactiques. Milchwissenschaft, 27, 503-507.

[16] Valles (E.) (1955). - Sur l'emploi de différents indicateurs d'oxido-réduction pour l'étude des bactériophages des streptocoques lactiques. Le Lait, $35,241-258$ 